\title{
Effects of Through-Flow and Internal Heat Generation on a Thermo Convective Instability in an Anisotropic Porous Medium
}

\author{
R. K. Vanishree \\ Maharani's Science college for Women, Bangalore - 560 001, India \\ Email:vanirkmscw@gmail.com
}

(Received December 31, 2012; accepted April 3, 2013)

\begin{abstract}
A linear stability analysis is performed to study the effects of through-flow and internal heat generation on the preferred mode of stationary thermal convection in a variable viscosity liquid saturating an anisotropic porous medium. The Rayleigh-Ritz technique is used to obtain the eigenvalue of the problem. The influence of porous parameter, mechanical anisotropy parameter, Peclet number, thermal anisotropy parameter, Brinkman number and variable viscosity parameter on the stability of the system is analyzed. The problem suggests another method of controlling convection by externally controlling porous media damping and shear. This is in addition to the through-flow mechanism of regulating convection.
\end{abstract}

Keywords: Anisotropy, Through-flow, Porous medium, Thermal convection, Internal heat generation.

\section{NOMENCLATURE}

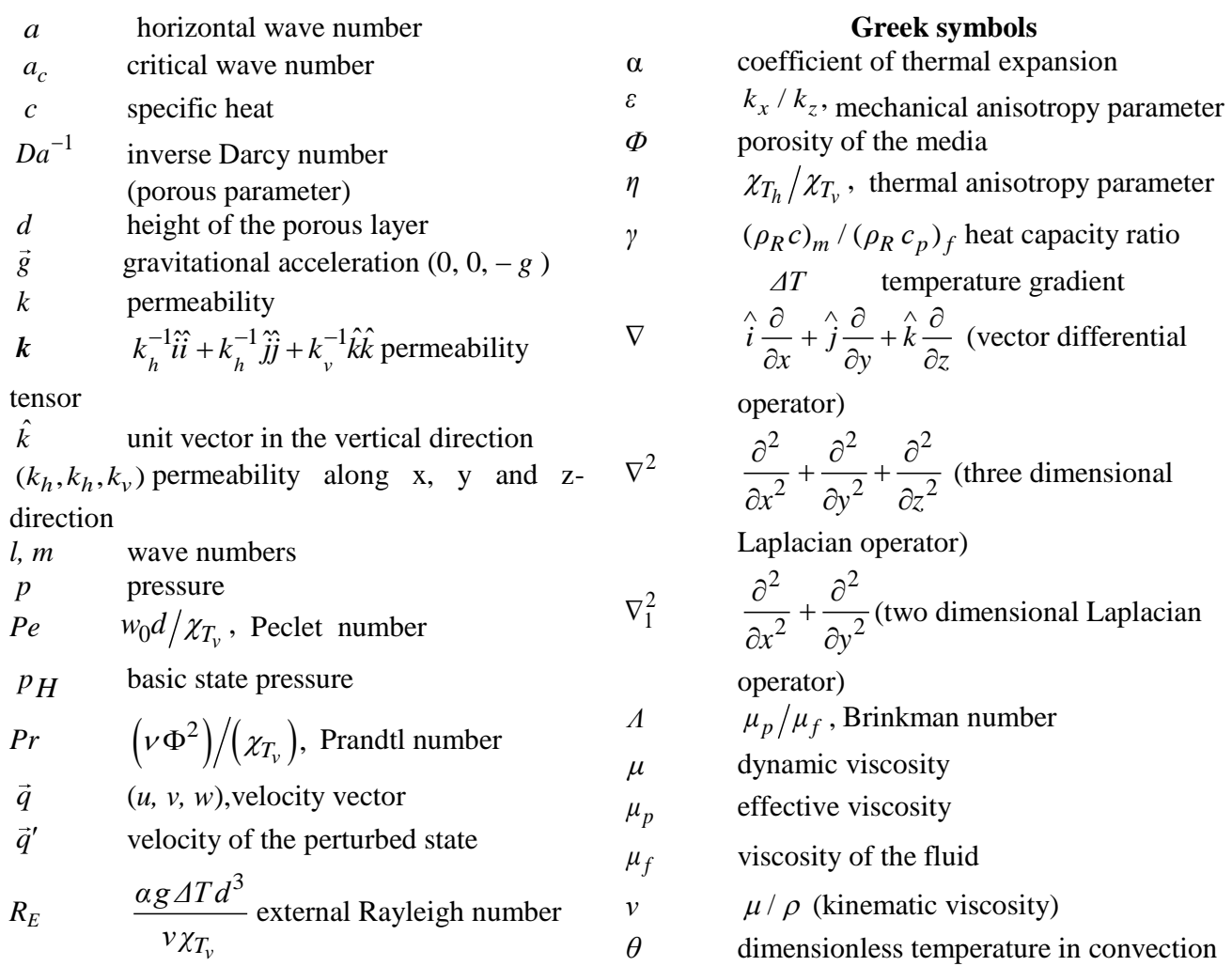




$\begin{array}{lll}R_{I} & \frac{\alpha g Q d^{5}}{v \chi_{T_{v}}}, \text { internal Rayleigh number } & x^{*}, z^{*} \\ t & \text { time } & \\ T & \text { temperature field } & \rho \\ T_{b} & \text { basic state temperature } & \rho_{b} \\ T_{R} & \text { reference temperature } & \rho_{R} \\ u, v, w & \text { dimensional horizontal and vertical } \\ & \text { velocity components } & b \\ u^{*}, v^{*}, w^{*} \text { dimensionless velocity components } & c \\ V_{1} & \text { linear variable viscosity parameter } & f \\ V_{2} & \text { quadratic variable viscosity parameter } & R \\ (x, y, z) & \text { Cartesian coordinates with z-axis } & , \\ & \text { vertically upwards } & *\end{array}$

\section{INTRODUCTION}

Thermal convection in horizontal fluid layer subject to constant but different temperatures at the boundaries has been extensively investigated by many researchers because of its importance in number of engineering and geophysical problems and also in energy storage applications. In these problems control of convective instability plays an important role. One of the effective mechanisms to control the convective instability is maintaining a non uniform temperature gradient across the fluid layer. In the classical Rayleigh-Bénard problem there is no flow of fluid across the horizontal boundaries. A modified problem, where the boundaries are permeable and there is vertical through-flow produced by injection at one end and removal of the fluid at the other, by uniformly distributed internal heat sources.

The effect of through-flow on the onset of convection in the Rayleigh-Bénard problem in a porous medium was studied by many authors. Rayleigh instability of a thermal boundary layer in flow through porous medium was studied by Wooding (1960). The through-flow effect on the convective instability in a fluid layer is investigated by Shvartsblat $(1968,1969)$ a because of the possibility of controlling the convective instability by adjusting the through-flow. Sutton (1970) studied the onset of convection in a porous channel with net through-flow. Homsy and Sherwood (1976) analyzed the convective instability in porous media with through-flow. The results of Shvartsblat were summarized by Gershuni and Zhukhovitzkii (1976) and it was concluded that the effect of through-flow is stabilizing independent of the direction of through-flow. Jones and Persichetti (1986) studied the convective instability in packed beds with through-flow. Nield (1987) has studied the effect of through-flow on Rayleigh-Bénard convection for rigid-rigid, free-free and rigid-free boundaries which are either insulating or conducting and has pointed out that the effect of through-flow is not always stabilizing. Suctioninjection effects on Rayleigh-Benard convection in a closely packed porous bed with general boundary condition on temperature was established by Siddheshwar (1995). Onset of convection in a

\author{
dimensionless horizontal and vertical \\ coordinate \\ density \\ basic state density \\ density of the liquid at reference temperature \\ $T=T_{R}$ \\ basic state \\ critical quantity \\ fluid \\ reference value \\ Superscripts \\ dimensional quantities \\ dimensionless quantities
}

porous layer with net through-flow and internal heat generation, stability of double diffusive convection in a porous layer with through-flow and the nonDarcian effects on the onset of convection in a porous layer with through-flow were studied by Khalili and Shivakumara (1998, 2001, 2003). Shivakumara (1999) analyzed the boundary and inertia effects on convection in porous media with through-flow. Effects of through-flow and internal heat generation on convective instabilities in an anisotropic porous layer and convective instability in superposed fluid and porous layers with vertical through-flow was studied by Khalili et al.(2002, 2003). Shivakumara and Nanjundappa (2006) analyzed the effects of quadratic drag and throughflow on double diffusive convection in a porous layer. Ansari (2007) studied the internal heat generation in a tall Cavity filled with a porous medium. Brevdo and Ruderman (2009) analyzed the convection in a porous medium with inclined temperature gradient and vertical throughflow. Barletta et. al. (2010) studied the convective roll instabilities of vertical throughflow with viscous dissipation in a horizontal porous layer. Onset of convection in a porous medium with strong vertical throughflow was investigated by Nield (2011).

Effect of radiation on non-Darcy free convection from a vertical cylinder embedded in a fluidsaturated porous medium with a temperaturedependent viscosity was studied by El-Hakiem and Rashad (2007). Effect of rotation on thermal convection in an anisotropic porous medium with temperature-dependent viscosity was investigated by Vanishree and Siddheshwar (2010). Veerraju et. Al. (2012) have studied the mixed convection at a vertical plate in a porous medium with magnetic field and variable viscosity. Convective instabilities in a variable-viscosity fluid with through-flow and internal heat generation in an anisotropic porous medium has not been given any attention. Hence in this paper we study the effect of through-flow and internal heat generation on convection in a variableviscosity liquid occupying an anisotropic porous medium. 


\section{MATHEMATICAL FORMULATION}

Consider a horizontal anisotropic porous layer of infinite extent occupied by a Newtonian fluid with temperature dependent viscosity, confined between stress free isothermal boundaries at $z=-d / 2$ and $z=$ $d / 2$ at which the temperatures are $T_{0}+\Delta T$ and $T_{0}$ respectively. A Cartesian co-ordinate system is taken with the origin mid-way between the two horizontal boundaries and $z$-axis vertically upwards. A constant vertical through-flow of magnitude $w_{0}$ is maintained at the boundaries. The porous medium is assumed to have high porosity and hence the fluid flow is governed by Brinkman model. An appropriate single-phase heat transport equation is chosen with effective heat capacity ratio, effective thermal diffusivity and internal heat generation. Thus the governing equations for the RayleighBénard situation in a Newtonian liquid with temperature-dependent viscosity occupying an anisotropic porous layer are:

Conservation of mass

$\nabla \cdot \vec{q}=0$

Conservation of linear momentum

$$
\begin{aligned}
& \rho_{R}\left[\frac{1}{\Phi} \frac{\partial \vec{q}}{\partial t}+\frac{1}{\Phi^{2}}(\vec{q} \cdot \nabla) \vec{q}\right]=-\nabla p+\rho \vec{g}-\frac{\mu_{f}}{k_{v}} \boldsymbol{k} \cdot \vec{q} \\
& +\nabla \cdot\left[\mu_{p}\left(\nabla \vec{q}+\nabla \vec{q}^{T r}\right)\right],
\end{aligned}
$$

Conservation of energy

$\gamma \frac{\partial T}{\partial t}+\vec{q} . \nabla T=\chi_{T v}\left[\eta\left(\frac{\partial^{2} T}{\partial x^{2}}+\frac{\partial^{2} T}{\partial y^{2}}\right)+\frac{\partial^{2} T}{\partial z^{2}}\right]+Q$,

Equation of state

$$
\rho=\rho_{R}\left[1-\alpha\left(T-T_{0}\right)\right],
$$

Thermorheological Relations

$$
\begin{aligned}
& \mu_{f}(T)=\mu_{1}\left[1-\Gamma_{1}\left(T-T_{0}\right)-\Gamma_{2}\left(T-T_{0}\right)^{2}\right] \\
& \mu_{p}(T)=\mu_{2}\left[1-\Gamma_{1}\left(T-T_{0}\right)-\Gamma_{2}\left(T-T_{0}\right)^{2}\right] .
\end{aligned}
$$

\section{BASIC STATE}

The aim of this paper is to investigate the stability of a quiescent state to infinitesimal perturbations superposed on the basic state. The basic state of the liquid being quiescent is described by

$\frac{\partial()}{\partial t}=0, \vec{q}_{b}=w_{0} \hat{k}, T=T_{b}(z), \rho=\rho_{b}(z)$,

$\mu_{f}=\mu_{f_{b}}(z), \mu_{p}=\mu_{p_{b}}(z)$.

The temperature $T_{b}$, pressure $p_{b}$ and density $\rho_{b}$ satisfy

$$
\begin{aligned}
& \frac{d p_{b}}{d z}=-\rho_{b} g, \\
& \frac{d^{2} T_{b}}{d z^{2}}-w_{0} \frac{d T_{b}}{d z}=-\frac{Q}{\chi T_{v}}, \\
& \rho_{b}=\rho_{R}\left[1-\alpha\left(T_{b}-T_{0}\right)\right], \\
& \mu_{f_{b}}=\mu_{1}\left[1-\Gamma_{1}\left(T_{b}-T_{0}\right)-\Gamma_{2}\left(T_{b}-T_{0}\right)^{2}\right],
\end{aligned}
$$

and

$$
\mu_{p_{b}}=\mu_{2}\left[1-\Gamma_{1}\left(T_{b}-T_{0}\right)-\Gamma_{2}\left(T_{b}-T_{0}\right)^{2}\right] .
$$

Solving Eq. (9) for $T_{b}$ subject to the following boundary conditions

$$
\begin{aligned}
& T_{b}=T_{0} \quad \text { at } \quad z=d / 2, \\
& T_{b}=T_{0}+\Delta T \quad \text { at } \quad z=-d / 2,
\end{aligned}
$$

we get

$T_{b}=T_{0}+\bar{\theta}_{b}(z)$,

where

$$
\bar{\theta}_{b}(z)=\frac{v \chi_{v}}{\alpha g d^{3}}\left(\begin{array}{c}
\frac{R_{I}}{P e}\left\{(z-1 / 2)-\left(\frac{e^{P e z}-e^{P e} / 2}{2 \sinh (P e / 2)}\right)\right\} \\
-R_{E}\left\{\frac{e^{P e z}-e^{P e} / 2}{2 \sinh (P e / 2)}\right\}
\end{array}\right) .
$$

\section{LINEAR STABILITY ANALYSIS}

Let the basic state be disturbed by an infinitesimal thermal perturbation. We now have $\vec{q}=w_{0} \hat{k}+\vec{q}^{\prime}, T=T_{b}(z)+\theta$,

$p_{b}=p_{b}(z)+p^{\prime}, \rho_{b}=\rho b(z)+\rho^{\prime}$,

$\mu_{f}=\mu_{f_{b}}(z)+\mu_{f}^{\prime}, \mu_{p}=\mu_{p_{b}}(z)+\mu_{p}^{\prime}$.

The prime indicates that the quantities are infinitesimal perturbations.

Substituting Eq. (15) into Eqs. (1) to (6) and using the basic state solution, we get the linearized equations governing the infinitesimal perturbations in the form:

$\nabla \cdot \vec{q}^{\prime}=0$,

$\rho_{R} w_{0} \frac{\partial \vec{q}^{\prime}}{\partial z}=-\nabla p^{\prime}+\alpha \rho_{R} g \theta \hat{k}-\frac{\mu f_{b}}{k_{v}} \boldsymbol{k} \cdot \vec{q}^{\prime}$

$+\nabla \mu_{p_{b}} \cdot\left(\nabla \vec{q}^{\prime}+\nabla \vec{q}^{T r}\right)+\mu_{p_{b}} \nabla^{2} \vec{q}^{\prime}$,

$w_{0} \frac{\partial \theta}{\partial z}+w^{\prime} \frac{\partial \overline{\theta_{b}}}{\partial z}=\chi_{T v}\left[\eta\left(\frac{\partial^{2} T^{\prime}}{\partial x^{2}}+\frac{\partial^{2} T^{\prime}}{\partial y^{2}}\right)+\frac{\partial^{2} T^{\prime}}{\partial z^{2}}\right]$,

$\rho^{\prime}=-\alpha \rho_{R} \theta$.

In writing the above equations we have assumed the principle of exchange of stabilities (PES) to be valid.

The perturbation Eqs. (16) to (19) are nondimensionalized using the following definitions

$$
\begin{aligned}
& \left(x^{*}, y^{*}, z^{*}\right)=\left(\frac{x}{d}, \frac{y}{d}, \frac{z}{d}\right), \\
& w^{*}=\frac{w^{\prime}}{\left(\begin{array}{l}
x \\
v / d
\end{array}\right)}, \theta^{*}=\frac{\theta}{\left(\begin{array}{c}
v x \\
v / \alpha g d^{3}
\end{array}\right)} .
\end{aligned}
$$


Using Eqs. (11), (12) and (19) in Eq. (17), operating curl twice on the resulting equation, and rendering this equation dimensionless, using Eq. (20), we get

$$
\begin{aligned}
& -\frac{\partial}{\partial z}\left(\nabla^{2} w\right)=-\frac{P r}{P e} \nabla_{1}^{2} \theta+\left[\begin{array}{c}
\frac{2 \Lambda P r}{P e} \frac{\partial}{\partial z}\left(\nabla^{2} w\right) \\
-\frac{D a^{-1} P r}{\varepsilon P e} \frac{\partial w}{\partial z}
\end{array}\right] \\
& \frac{V_{1}}{R_{E}} P_{1}(z)+\left[\begin{array}{c}
\frac{4 \Lambda P r}{P e} \frac{\partial}{\partial z}\left(\nabla^{2} w\right) \\
-\frac{2 D a^{-1} P r}{\varepsilon P e} \frac{\partial w}{\partial z}
\end{array}\right] \frac{V_{2}}{R_{E}^{2}} P_{2}(z) \\
& +\left[\begin{array}{r}
\frac{D a^{-1} P r}{P e}\left(\nabla_{1}^{2} w+\frac{1}{\varepsilon} \frac{\partial^{2} w}{\partial z^{2}}\right) \\
-\frac{\Lambda P r}{P e} \nabla^{4} w
\end{array}\right]\left[\begin{array}{c}
1-\frac{V_{1}}{R_{E}} P_{3}(z) \\
-\frac{V_{2}}{R_{E}^{2}} P_{4}(z)
\end{array}\right] \\
& +\frac{\Lambda P r V}{R_{E}} \frac{R_{E} P e+R_{I}}{2 \sinh (P e / 2)} e^{P e z}\left(\nabla_{1}^{2} w-\frac{\partial^{2} w}{\partial z^{2}}\right) \\
& \left.-\frac{2 \Lambda P r}{P e} \frac{V_{2}}{R_{E}^{2}}\left(\nabla_{1}^{2} w-\frac{\partial^{2} w}{\partial z^{2}}\right) P_{5}(z)\right)
\end{aligned}
$$

$\eta \nabla_{1}^{2} \theta+\frac{\partial^{2} \theta}{\partial z^{2}}-P e \frac{\partial \theta}{\partial z}=w\left[\frac{R_{I}}{P e}-\frac{R_{E} P e+R_{I}}{2 \sinh (P e / 2)} e^{P e z}\right]$,

where

$$
\begin{aligned}
& P_{1}(z)=\frac{R_{I}}{P e}-\frac{R_{E} P e+R_{I}}{2 \sinh (P e / 2)} e^{P e z}, \\
& P_{2}(z)=\left[\begin{array}{l}
\frac{R_{I}^{2}}{P e^{2}}(z-1 / 2)-\frac{R_{I}\left(R_{E} P e+R_{I}\right)}{2 P e \sinh (P e / 2)} e^{P e z}(z-1 / 2) \\
-\frac{R_{I}\left(R_{E} P e+R_{I}\right)}{2 P e^{2} \sinh (P e / 2)}\left(e^{P e z}-e^{P e} / 2\right) \\
+\frac{\left(R_{E} P e+R_{I}\right)^{2}}{4 P e \sinh ^{2}(P e / 2)} e^{P e z}\left(e^{P e z}-e^{P e} / 2\right)
\end{array}\right], \\
& P_{3}(z)=\frac{R_{I}}{P e}(z-1 / 2)-\frac{R_{E} P e+R_{I}}{2 \sinh (P e / 2)}\left(e^{P e z}-e^{P e / 2}\right),
\end{aligned}
$$

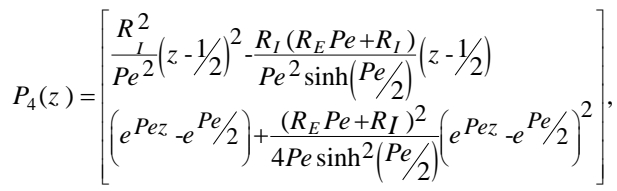

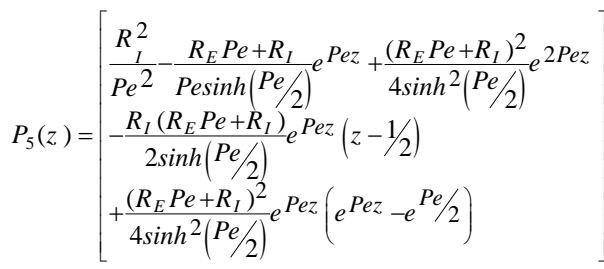

The asterisks have been dropped for simplicity in the above equations, the nondimensional parameters in the above equations are: $\operatorname{Pr}$, Prandtl number, $R_{E}$, thermal Rayleigh number, $R_{I}$, internal Rayleigh number, $P e$, Peclet number, $\varepsilon$, mechanical anisotropy parameter, $\eta$, thermal anisotropy parameter, $D a^{-1}$, inverse Darcy number, $\Lambda$, Brinkmann number, $V_{1}$, linear variable viscosity parameter, and $V_{2}$, quadratic variable viscosity parameter. Eqs. (21) and (22) are two equations in two unknowns, $w$ and $\theta$.

Equations (21) and (22) are to be solved subject to the conditions

$w=\nabla^{2} w=\theta=0$ at $z= \pm 1 / 2$

The infinitesimal perturbations $w$ and $\theta$ are assumed to be periodic waves (see Chandrasekhar, 1961) and hence these permit normal mode solution in the form

$\left[\begin{array}{l}w \\ \theta\end{array}\right]=\left[\begin{array}{l}w(z) \\ \theta(z)\end{array}\right] e^{i(l x+m y)}$,

where $w(z)$ and $\theta(Z)$ are the amplitudes and $l$ and $m$ are the components of the wave number vector. These satisfy the boundary conditions of equations (23). We also document here the fact that asymmetric boundary conditions have been chosen here to bring in the thermorheological effect in all the cases of interest that can be got as a limiting case of the present study. Substituting Eq. (24) in Eqs. (21) and (22), we get

$$
\begin{aligned}
& {\left[\begin{array}{c}
\frac{1}{R_{E}}\left\{F_{1}(D)\right\}+\frac{1}{R^{2}}\left\{F_{2}(D)\right\} \\
+\frac{\left(R_{E} P e+R_{I}\right)}{R_{E}}\left\{F_{3}(D)\right\} \\
+\frac{\left(R_{E} P e+R_{I}\right)}{R_{E}^{2}}\left\{F_{4}(D)\right\} \\
+\frac{\left(R_{E} P e+R_{I}\right)^{2}}{R_{E}^{2}}\left\{F_{5}(D)\right\} \\
+C_{13}\left\{F_{6}(D)\right\}-C_{19}\left\{F_{7}(D)\right\} \\
++\left\{F_{8}(D)\right\}
\end{array}\right] w+C_{31} \theta=0,(25)} \\
& {\left[\begin{array}{c}
\left.\frac{R_{I}}{P e}-\frac{R_{E} P e+R_{I}}{2 \sinh (P e} / 2\right) \\
e^{P e z}
\end{array}\right] w+\left[-D^{2}+D+\eta a^{2}\right] \theta=0}
\end{aligned}
$$

where

$$
\begin{aligned}
& F_{1}(D)=C_{1}\left(D^{3}-a^{2} D\right)-C_{3}(D)-C_{14}(z-1 / 2)\left(D^{2}-\varepsilon a^{2}\right) \\
& +C_{20}(z-1 / 2)\left(D^{2}-a^{2}\right)^{2}, \\
& F_{2}(D)=C_{5}(z-1 / 2)\left(D^{3}-a^{2} D\right)-C_{9}(z-1 / 2) D \\
& -C_{16}(z-1 / 2)^{2}\left(D^{2}-\varepsilon a^{2}\right)+C_{22}(z-1 / 2)^{2}\left(D^{2}-a^{2}\right)^{2}, \\
& F_{3}(D)=-C_{2} e^{P e z}\left(D^{3}-a^{2} D\right)+C_{4}\left(e^{P e z} D\right) \\
& +C_{15}\left(e^{P e z}-e^{P e} / 2\right)\left(D^{3}-a^{2} D\right) \\
& -C_{21}\left(e^{P e z}-e^{P e} / 2\right)\left(D^{2}-a^{2}\right)^{2}-C_{25} e^{P e z}\left(D^{2}+a^{2}\right), \\
& F_{4}(D)=-C_{6} e^{P e z}(z-1 / 2)\left(D^{3}-a^{2} D\right)-C_{7}\left(e^{P e z}-e^{P e} / 2\right) \\
& \left(D^{3}-a^{2} D\right)+C_{10} e^{P e z}(z-1 / 2) D+C_{11}\left(e^{P e z}-e^{P e} / 2\right) D \\
& +C_{17}(z-1 / 2)\left(e^{P e z}-e^{P e} / 2\right)\left(D^{2}-\varepsilon a^{2}\right)-C_{23}(z-1 / 2) \\
& \left(e^{P e z}-e^{P e} / 2\right)\left(D^{2}-a^{2}\right)^{2}-C_{27} e^{P e z}\left(D^{2}+a^{2}\right) \\
& -C_{29} e^{P e z}(z-1 / 2)\left(D^{2}+a^{2}\right),
\end{aligned}
$$




$$
\begin{aligned}
& F_{5}(D)=C_{8} e^{P e z}\left(e^{P e z}-e^{P e} / 2\right)\left(D^{3}-a^{2} D\right)-C_{12} e^{P e z} \\
& \left(e^{P e z}-e^{P e} / 2\right) D-C_{18}\left(e^{P e z}-e^{P e} / 2\right)^{2}\left(D^{2}-\varepsilon a^{2}\right) \\
& +C_{24}\left(e^{P e z}-e^{P e} / 2\right)^{2}\left(D^{2}-a^{2}\right)+C_{28} e^{2 P e z}\left(D^{2}+a^{2}\right) \\
& +C_{30} e^{P e z}\left(e^{P e z}-e^{P e} / 2\right)\left(D^{2}+a^{2}\right) \\
& F_{6}(D)=\left(D^{2}-\varepsilon a^{2}\right), F_{7}(D)=\left(D^{2}-a^{2}\right)^{2}, \\
& F_{8}(D)=\left(D^{3}-a^{2} D\right) \\
& C_{1}=\frac{2 \Lambda P r V_{1} R_{I}}{P e^{2}}, C_{2}=\frac{\Lambda P r V_{1}}{\operatorname{Pesinh}(P e / 2)}, \\
& C_{3}=\frac{D a^{-1} \operatorname{PrV}_{1} R_{I}}{\varepsilon P e^{2}}, C_{4}=\frac{D a^{-1} \operatorname{Pr} V_{1}}{2 \varepsilon \operatorname{Pesinh}(P e / 2)}, \\
& C_{5}=\frac{4 \Lambda \operatorname{PrV}_{2} R_{I}^{2}}{P e^{3}}, C_{6}=\frac{4 \Lambda \operatorname{PrV}_{2} R_{I}}{2 P e^{2} \sinh (P e / 2)}, \\
& C_{7}=\frac{2 \Lambda \operatorname{PrV}_{2} R_{I}}{P e^{3} \sinh \frac{P e}{2}}, C_{8}=\frac{\Lambda P r V_{2}^{2}}{P e^{2} \sinh ^{2}(P e / 2)}, \\
& C_{9}=\frac{D a^{-1} P_{2} V_{2} R_{I}^{2}}{\varepsilon P e^{3}}, C_{10}=\frac{D a^{-1} P r V_{2}}{\varepsilon P e^{2} \sinh (P e / 2)}, \\
& C_{11}=\frac{D a^{-1} \operatorname{PrV}_{2} R_{I}}{\varepsilon P e^{3} \sinh (P e / 2)}, C_{12}=\frac{D a^{-1} P r V_{2}}{2 \varepsilon P e^{2} \sinh ^{2}(P e / 2)}, \\
& C_{13}=\frac{D a^{-1} P r}{\varepsilon P e}, C_{14}=C_{3}, C_{15}=\frac{D a^{-1} P r V_{1}}{2 \varepsilon P e^{2} \sinh (P e / 2)}, \\
& C_{16}=\frac{C_{9}}{2}, C_{17}=C_{11}, C_{18}=\frac{D a^{-1} P r V_{2}}{4 \varepsilon P e^{3} \sinh (P e / 2)}, \\
& C_{19}=\frac{\Lambda P r}{P e}, C_{20}=\frac{\Lambda P r V_{1} R_{I}}{P e^{2}}, C_{21}=\frac{\Lambda P r V_{1}}{2 P e^{2} \sinh (P e / 2)}, \\
& C_{22}=\frac{C_{5}}{4}, C_{23}=\frac{C_{7}}{2}, C_{24}=\frac{\Lambda \operatorname{PrV}_{2}}{4 P e^{3} \sinh ^{2}(P e / 2)}, \\
& C_{25}=\frac{\Lambda P r V_{1}}{2 \sinh (P e / 2)}, C_{26}=\frac{C_{5}}{2}, C_{27}=\frac{\Lambda \operatorname{PrR}_{I}}{P e^{2} \sinh (P e / 2)}, \\
& C_{28}=\frac{\Lambda \operatorname{PrV}_{2}}{2 \operatorname{Pesinh}^{2}(\mathrm{Pe} / 2)}, C_{29}=\frac{\Lambda \operatorname{PrV}_{2} R_{I}}{\operatorname{Pesinh}(\mathrm{Pe} / 2)}, \\
& C_{30}=C_{28}, C_{31}=\frac{P r}{P e} \text {. }
\end{aligned}
$$

The set of ordinary differential Eqs. (25) and (26) are approximations based on physical considerations to the system of partial differential Eqs. (21) and (22). Although the relationship between the solutions of the governing partial differential equations and the corresponding ordinary differential equations has not been established, these linear models reproduce qualitatively the convective phenomena observable through the full system.

\section{APPLICATION of RAYLEIGH- RITZ TECHNIQUE}

In this section we apply the Rayleigh-Ritz method to Eqs. (25) and (26) that gives general results on the eigenvalue of the problem using the trial functions for the lowest eigenvalue. We obtain an approximate solution of the differential equations with the given boundary conditions by choosing

trial functions for velocity and temperature perturbations that may satisfy the boundary conditions but may not exactly satisfy the differential equations. This leads to residuals when the trial functions are substituted into the differential equations. The Rayleigh-Ritz method requires the residual to be orthogonal to each individual trial function. In the Rayleigh-Ritz procedure, we expand the velocity and temperature by

$$
\begin{aligned}
& w(z, t)=\sum A_{i}(t) w_{i}(z), \\
& \theta(z, t)=\sum B_{i}(t) \theta_{i}(z),
\end{aligned}
$$

where $w_{i}(z)$ and $\theta_{i}(z)$ are trial functions that have to satisfy the boundary conditions. For the purpose of illustration we present below the single-term Rayleigh-Ritz technique. In obtaining the eigenvalue, however, we have used the higher order method with the number of terms used depending on the parameter values.

Multiplying Eq. (25) by $w$ and Eq. (26) by $\theta$, integrating the resulting equation by parts with respect to $z$ from $-1 / 2$ to $1 / 2$ and taking $w=A w_{1}$ and $\theta=B \theta_{1}$ in which $A$ and $B$ are constants and $w_{1}$ and $\theta_{1}$ are trial functions. This procedure yields the following cubic equation, the least positive root of which is the lowest Rayleigh number:

$G_{1} R_{E}^{3}+G_{2} R_{E}^{2}+G_{3} R_{E}+G_{4}=0$,

where

$$
\begin{aligned}
& G_{1}=\frac{C_{31} D_{31} E_{1} P e}{2 \sinh (P e / 2)}, G_{2}=\left(\begin{array}{c}
\left(P e^{2} F_{1}+P e F_{2}+F_{3}\right)\left(\begin{array}{l}
-E_{2}+P e E_{3} \\
+\eta a^{2} E_{4}
\end{array}\right) \\
-C_{31} D_{31}\left(\frac{R_{I} D_{31}}{P e}-\frac{R_{I} E_{1}}{2 \sinh (P e / 2)}\right)
\end{array}\right), \\
& G_{3}=\left(2 P e R_{I} F_{1}+R_{I} F_{2}+P e F_{4}+F_{5}\right)\left(-E_{2}+P e E_{3}+\eta_{1} a^{2} E_{4}\right) \text {, } \\
& G_{4}=\left(R_{I}^{2} F_{1}+R_{I} F_{4}+F_{6}\right)\left(-E_{2}+P e E_{3}+\eta_{1} a^{2} E_{4}\right) \text {, } \\
& D_{1}=\left\langle w_{1} D^{3} w_{1}\right\rangle-a^{2}\left\langle w_{1} D w_{1}\right\rangle,
\end{aligned}
$$

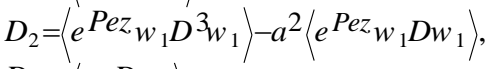

$$
\begin{aligned}
& D_{3}=\left\langle w_{1} D w_{1}\right\rangle \text {, } \\
& D_{4}=\left\langle e^{P e z_{1}} w_{1} D w_{1}\right\rangle, D_{5}=\left(\begin{array}{l}
\left\langle(z-1 / 2) w_{1} D^{3} w_{1}\right\rangle \\
-a^{2}\left\langle(z-1 / 2) w_{1} D w_{1}\right\rangle
\end{array}\right), \\
& D_{6}=\left(\begin{array}{c}
\left\langle e^{P e z}(z-1 / 2) w_{1} D^{3} w_{1}\right\rangle \\
-a^{2}\left\langle e^{P e z}(z-1 / 2) w_{1} D w_{1}\right\rangle
\end{array}\right), \\
& D_{7}=\left(\begin{array}{c}
\left\langle\left(e^{P e z}-e^{P e / 2}\right) w_{1} D^{3} w_{1}\right\rangle \\
-a^{2}\left\langle\left(e^{P e z}-e^{P e} / 2\right) w_{1} D w_{1}\right\rangle
\end{array}\right), \\
& D_{8}=\left(\begin{array}{c}
\left\langle e^{P e z}\left(e^{P e z}-e^{P e} / 2\right) w_{1} D^{3} w_{1}\right\rangle \\
-a^{2}\left\langle e^{P e z}\left(e^{P e z}-e^{P e} / 2\right) w_{1} D w_{1}\right\rangle
\end{array}\right),
\end{aligned}
$$




$$
\begin{aligned}
& D_{9}=\left\langle(z-1 / 2) w_{1} D w_{1}\right\rangle, D_{10}=\left\langle e^{P e z}(z-1 / 2) w_{1} D w_{1}\right\rangle, \\
& D_{11}=\left\langle\left(e^{P e z}-e^{P e} / 2\right) w_{1} D w_{1}\right\rangle, \\
& D_{12}=\left\langle e^{P e z}\left(e^{P e z}-e^{P e} / 2\right) w_{1} D w_{1}\right\rangle, \\
& D_{13}=\left\langle w_{1} D^{2} w_{1}\right\rangle-\varepsilon a^{2}\left\langle w_{1}^{2}\right\rangle \text {, } \\
& D_{14}=\left(\begin{array}{c}
\left\langle(z-1 / 2)^{w}{ }_{1} D^{2} w_{1}\right\rangle \\
-\varepsilon a^{2}\left\langle(z-1 / 2) w_{1}^{2}\right\rangle
\end{array}\right), \\
& D_{15}=\left(\begin{array}{c}
\left\langle\left(e^{P e z}-e^{P e} / 2\right) w_{1} D^{2} w_{1}\right\rangle \\
-\varepsilon a^{2}\left\langle\left(e^{P e z}-e^{P e} / 2\right) w_{1} D w_{1}\right\rangle
\end{array}\right), \\
& D_{16}=\left\langle(z-1 / 2)^{2} w_{1} D^{2} w_{1}\right\rangle-\varepsilon a^{2}\left\langle(z-1 / 2)^{2} w_{1}^{2}\right\rangle, \\
& D_{17}=\left(\begin{array}{c}
\left\langle(z-1 / 2)\left(e^{P e z}-e^{P e} / 2\right) w_{1} D^{2} w_{1}\right\rangle \\
\left.-\varepsilon a^{2}\left\langle(z-1 / 2)\left(e^{P e z}-e^{P e} / 2\right) w_{1}^{2}\right\rangle\right)
\end{array}\right), \\
& D_{18}=\left(\begin{array}{c}
\left(\left(e^{P e z}-e^{P e} / 2\right)^{2} w_{1} D^{2} w_{1}\right\rangle \\
-\varepsilon a^{2}\left\langle\left(e^{P e z}-e^{P e} / 2\right)^{2} w_{1}^{2}\right\rangle
\end{array}\right), \\
& D_{19}=\left\langle w_{1} D^{4} w_{1}\right\rangle+a^{4}\left\langle w_{1}^{2}\right\rangle-2 a^{2}\left\langle w_{1} D^{2} w_{1}\right\rangle, \\
& D_{20}=\left(\begin{array}{c}
\left\langle(z-1 / 2) w_{1} D^{4} w_{1}\right\rangle+a^{4}\left\langle\langle z-1 / 2)^{2}\right\rangle \\
-2 a^{2}\left\langle(z-1 / 2) w_{1} D^{2} w_{1}\right\rangle
\end{array}\right), \\
& D_{21}=\left(\begin{array}{c}
\left\langle\left(e^{P e z}-e^{P e} / 2\right) w_{1} D^{4} w_{1}\right\rangle \\
+a^{4}\left\langle\left(e^{P e z}-e^{P e} / 2 w_{1}^{2}\right\rangle\right. \\
-2 a^{2}\left\langle\left(e^{P e z}-e^{P e} / 2 w_{1} D^{2} w_{1}\right\rangle\right.
\end{array}\right),
\end{aligned}
$$

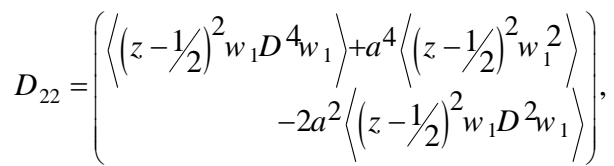

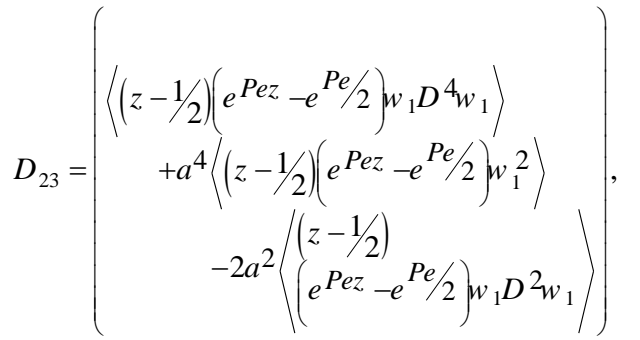

$$
\begin{aligned}
& D_{25}=\left\langle e^{P e z_{1}} w_{1} w_{1}\right\rangle+a^{2}\left\langle e^{P e z_{1}} w_{1}^{2}\right\rangle \text {, } \\
& D_{26}=\left\langle w_{1} D^{2} w_{1}\right\rangle+a^{2}\left\langle w_{1}^{2}\right\rangle, D_{27}=D_{25} \text {, } \\
& D_{28}=\left\langle e^{2 P e z_{w}} w_{1} D^{2} w_{1}\right\rangle+a^{2}\left\langle e^{2 P e z_{1}} w_{1}^{2}\right\rangle, \\
& D_{29}=\left\langle e^{P e z}(z-1 / 2) w_{1} D^{2} w_{1}\right\rangle+a^{2}\left\langle e^{P e z}(z-1 / 2) w_{1}^{2}\right\rangle \text {, }
\end{aligned}
$$

$$
\begin{aligned}
& D_{30}=\left(\begin{array}{c}
\left\langle e^{P e z}\left(e^{P e z}-e^{P e} / 2\right) w_{1} D^{2} w_{1}\right\rangle \\
-a^{2}\left\langle e^{P e z}\left(e^{P e z}-e^{P e} / 2\right) w_{1}^{2}\right\rangle
\end{array}\right), \\
& D_{31}=\left\langle w_{1} \theta_{1}\right\rangle, E_{1}=\left\langle e^{P e z} w_{1} \theta_{1}\right\rangle, E_{2}=\left\langle\theta_{1} D^{2} \theta_{1}\right\rangle, \\
& E_{3}=\left\langle\theta_{1} D \theta_{1}\right\rangle, E_{4}=\left\langle\theta_{1}^{2}\right\rangle, \\
& F_{1}=\left(\begin{array}{c}
C_{8} D_{8}-C_{12} D_{12}-C_{18} D_{18}+C_{24} D_{24} \\
+C_{28} D_{28}+C_{30} D_{30}
\end{array}\right), \\
& F_{2}=\left(\begin{array}{c}
-C_{2} D_{2}+C_{4} D_{4}+C_{15} D_{15} \\
-C_{21} D_{21}-C_{25} D_{25}
\end{array}\right), \\
& F_{3}=C_{13} D_{13}-C_{19} D_{19}+D_{1}, \\
& F_{4}=\left(\begin{array}{c}
-C_{6} D_{6}-C_{7} D_{7}+C_{10} D_{10} \\
+C_{11} D_{11}+C_{17} D_{17} \\
-C_{23} D_{23}-C_{27} D_{27}-C_{29} D_{29}
\end{array}\right), \\
& F_{5}=C_{1} D_{1}-C_{3} D_{3}-C_{14} D_{14}+C_{20} D_{20}, \\
& F_{6}=\left(\begin{array}{c}
C_{5} D_{5}-C_{9} D_{9}-C_{16} D_{16} \\
+C_{22} D_{22}+C_{26} D_{26}
\end{array}\right) .
\end{aligned}
$$

In the higher-order method we obtain higher-degree equations for the eigenvalue and the minimum positive root of this equation gives the critical eigenvalue. The corresponding wave number is the critical wave number.

\section{RESULTS AND DISCUSSIONS}

As made out in the introduction of the paper, there are numerous papers considering the individual effects of i) through flow, ii) temperaturedependent viscosity, iii) anisotropy and iv) internal heat generation. In the present paper a numerical study is made of the combination of the above effects on the onset of convection in a fluidsaturated anisotropic porous medium. These four effects are, respectively, represented by the Peclet number $P e$, the thermorheological parameters (or the variable viscosity parameters) $V_{1}$ and $V_{2}$, the anisotropy parameters $(\varepsilon, \eta)$ and the internal Rayleigh number $R_{I}$. The effect of the porous structure comes into play through the Darcy number $D a^{-1}$. Figures 2 to 13 are plots of critical Rayleigh number, $R_{c}^{s}$ and the critical wave number, $a_{c}^{s}$ versus the quadratic variable-viscosity parameter $V_{2}$ for stationary mode of convection. Before embarking on a discussion of the results depicted by the Figs. 2 to 13, we note that the principle of exchange of stabilities (PES) is valid in the problem. The higher order Rayleigh-Ritz method is used to obtain the critical values of Rayleigh number and wave number as a function of inverse Darcy number, internal Rayleigh number, Peclet number, thermal and mechanical anisotropy parameters, Prandtl number, linear and quadratic thermorheological parameters and the Brinkman number. It is also important to note in this problem that the Prandtl number influences the onset of stationary convection only due to the through-flow effect being there. 
Before discussing the results arrived at in the problem it is also important to observe that the basic state temperature distribution $\bar{\theta}_{b}(z)$ is nonlinear which throws light on the effect of heat source (sink) on the stability. We also note here that $\bar{\theta}_{b}(z)$ is independent of the mechanical anisotropy parameter. The distribution $\theta_{b}^{*}(z)$ being concave or convex depends on the value of $R_{I}, R_{E}$ and $P e$ (Khalili et al., 2002). The nature of this non-linear temperature distribution significantly matters in analyzing the parameter's influences on convection as this changes the energy supply to the disturbance. Khalili et al. (2002) gives a good account of the above three parameter influences on the scaled temperature distribution.

The porous medium considered is sparsely packed and hence $D a^{-1}$ is assumed to take the values from 1 to 100 . Figure 2 reveals that the effect of increasing quadratic thermo-rheological parameter $V_{2}$ is to destabilize the system and the effect of $D a^{-1}$ is to increase $R_{c}^{s}$. this result is in tune with intuition. Figure 3 illustrates that the effect of increasing $D a^{-1}$ is to decrease the cell size at the onset of convection, there by implying that the effect of porous parameter is to delay the onset of convection. One another fact that must be noted in this context is that in the absence of the parameter $V_{2}$, i.e., when the viscosity-temperature dependence is linear, the effect of increasing $V_{1}$ is to stabilize convection! This is against intuition. In view of this the viscosity-temperature relationship has been taken to be quadratic. In the strictest modeling of the viscosity-temperature relationship, the same has to be exponential. Computations effected by us revealed that for an exponential relationship higher-order method is impossible to implement on the computer systems that are available to us in the department.

The effect of the internal Rayleigh number $R_{I}$, for both heat source and heat sink cases, on the eigenvalue as a function of $V_{2}$ is shown in Fig. 4. Quite interestingly it is found that the effect of increasing $R_{I}$, from $-\mathrm{ve}$ to $+\mathrm{ve}$ is to stabilize the system for values of $V_{2}$ less than 0.4 . The reverse of this is seen for values of $V_{2}$ greater than o.6. Between 0.4 and 0.6 there is a pinch which implies that the effect of $R_{I}$ on $R_{c}^{s}$, in this range is quite small compared to its effect in other ranges. The effect of increasing $R_{I}$ on $a_{c}^{s}$ is to decrease the cell size. This is shown in Fig. 5.

Both pro-gravity $(\mathrm{Pe}<0)$ and anti-gravity $(\mathrm{Pe}>0)$ situation are covered in Figs. 6 and 7. The effect of increasing $|P e|$ is to stabilize the convection. Quite obviously from the figure the case $P e>0$ is more destabilizing compared to $P e<0$. This result is at variance with existing results on through-flow and possibly due to the temperature dependence of viscosity. The non-coincidence of the graphs in the two cases is indicative of the fact that $R_{c}^{S}$ is not an even function of $P e$. It is well known now that $R_{c}^{s}$ can be an even function of $P e$ only when we have symmetry in the system (Nield, 1987). In Fig. 6 one more important observation to make is that the slope of the $R_{c}^{s}$ Vs. $V_{2}$ curve is steeper for the case $P e>0$ compared to those of the case $P e<0$. Fig. 7 shows the effect of increasing $P e$ is to diminish the cell size at the onset of convection.

A mild anisotropy is considered and hence the values of mechanical and thermal anisotropy parameters $\varepsilon$ and $\eta$ are chosen around one $(\varepsilon=\eta$ $=1$ represent the isotropic media). The effect of increasing on $R_{c}^{s}$. is shown in Fig. 8. The effect of increasing $\varepsilon$ is obviously to decrease the cell size at the onset of convection (see Fig.9. The opposite behaviour is seen with increasing value of $\eta$ and this is shown in Fig.10. Figure 11 demonstrates the effect of $\eta$ on $a_{c}^{s}$ to be similar to that of $\varepsilon$. The results are in agreement with existing results. The effect of increasing Brinkman number $\Lambda$ on $R_{c}^{S}$ is shown to be to increase $R_{c}^{S}$ and the same is shown in Fig. 12. $\Lambda$ can assume a range of values that are greater than, equal to or less than 1 (see Givler and Altobelli, 2010). The effect of increasing $\Lambda$ on $a_{c}^{s}$ is opposite to that of $\varepsilon$ and $\eta$ and the same is shown in Fig. 13 In the limit of $V_{2}$ tending to zero, we recover the results of Khalili et al.(2002).

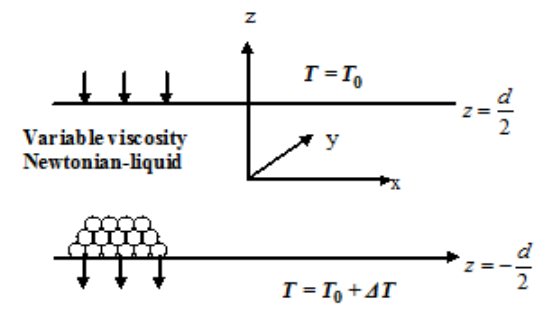

Fig. 1.a. Physical configuration for downward through-flow.

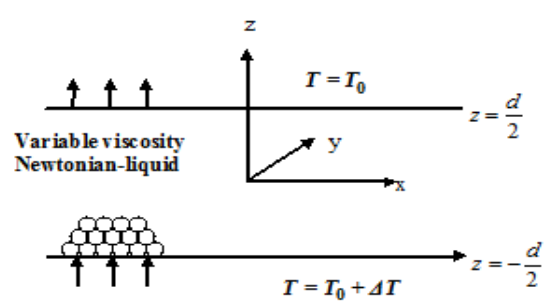

Fig. 1.b. Physical configuration for upward through-flow. 
R. K. Vanishree / JAFM, Vol. 7, No. 4, pp. 581-590, 2014.

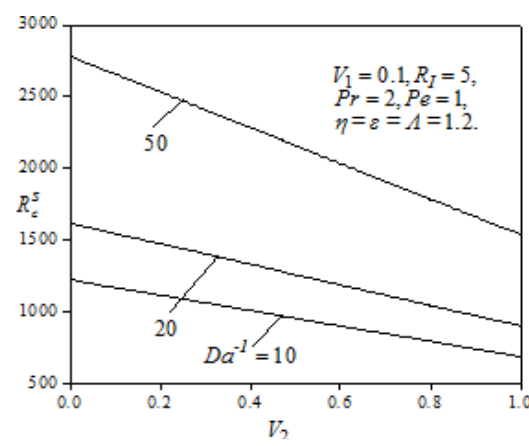

Fig. 2. Plot of critical Rayleigh number $R_{c}^{s}$ (stationary) Vs. quadratic variable viscosity parameter $V_{2}$ for different values of porous parameter $\mathrm{Da}^{-1}$.

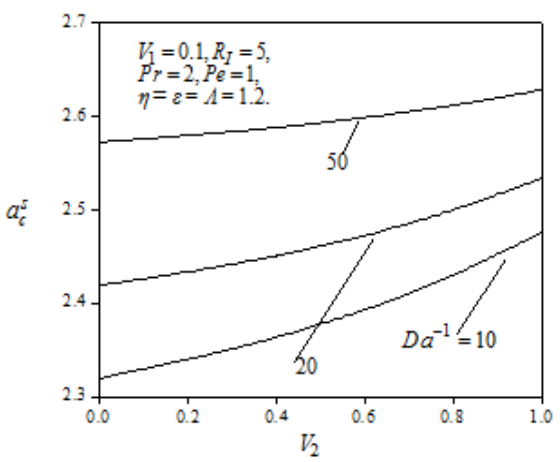

Fig. 3. Plot of critical wave number $a_{c}^{s}$ (stationary) Vs. $V_{2}$ for different values of $\mathrm{Da}^{-1}$.

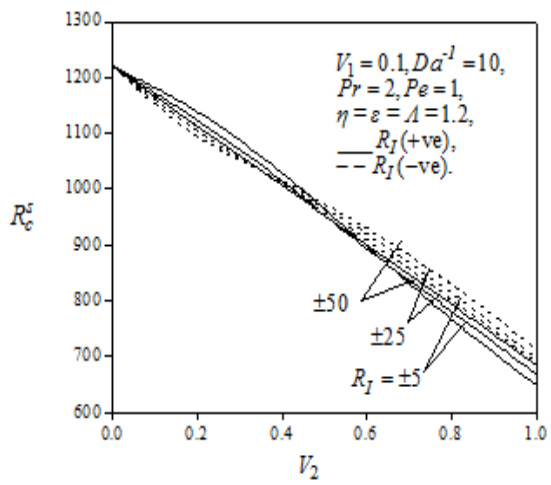

Fig. 4. Plot $R_{c}^{s}$ (stationary) Vs. $V_{2}$ for different values of internal Rayleigh number $R_{I}$.

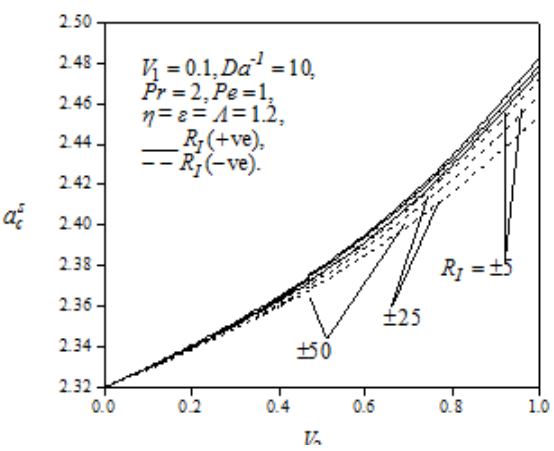

Fig. 5. Plot of $a_{c}^{s}$ Vs. $V_{2}$ for different values of $\boldsymbol{R}_{\boldsymbol{I}}$.

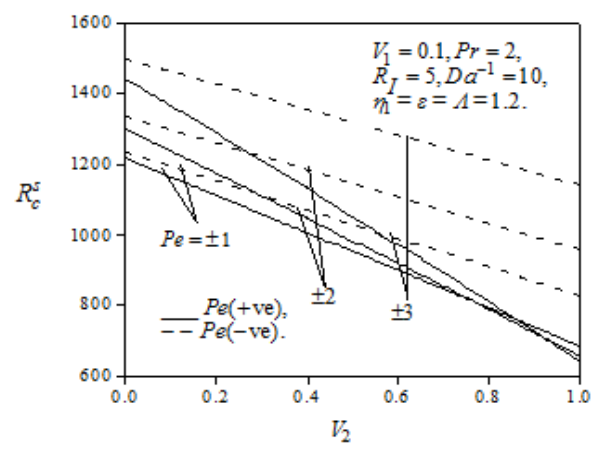

Fig. 6. Plot of $R_{c}^{s}$ Vs. $V_{2}$ for different values of Peclet number $P e$.

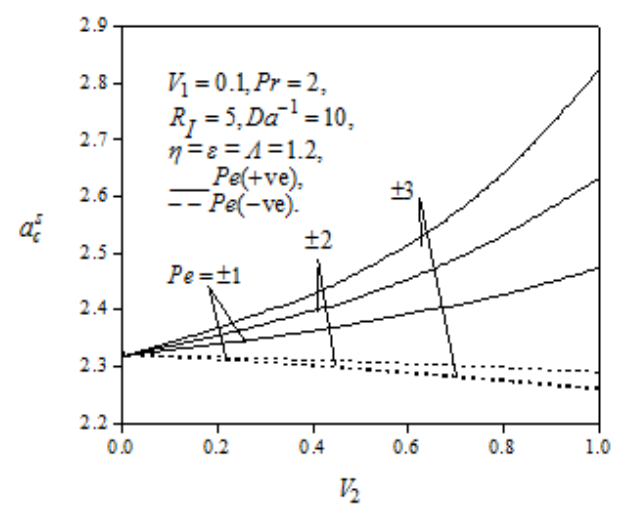

Fig. 7. Plot of $a_{c}^{s}$ Vs. $V_{2}$ for different values of $P e$.

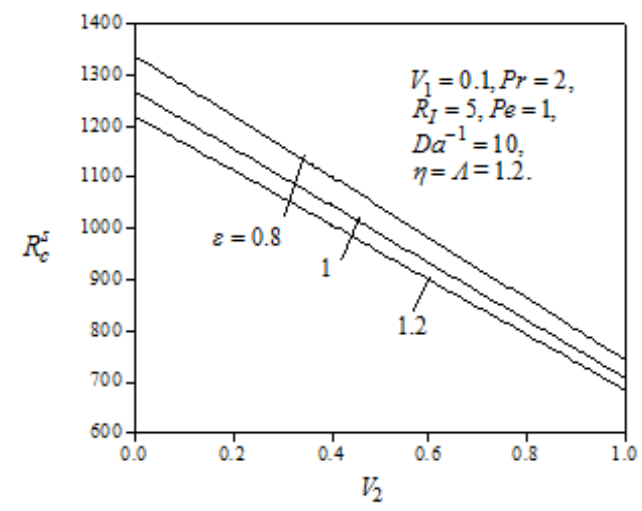

Fig. 8. Plot of $R_{c}^{s}$ Vs. $V_{2}$ for different values of mechanical anisotropy parameter $\varepsilon$.

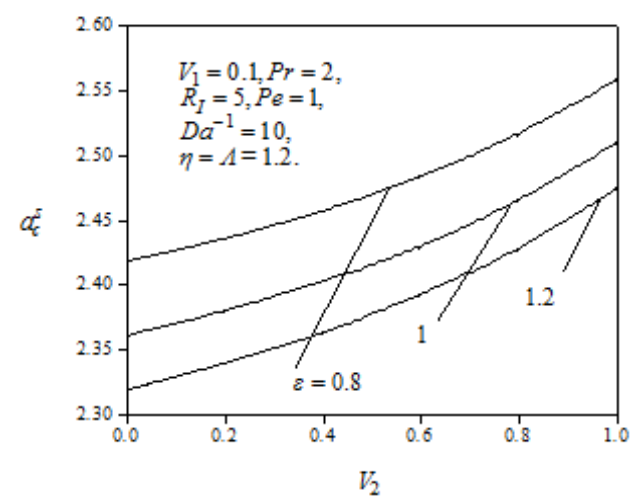

Fig. 9. Plot of $a_{c}^{s}$ Vs. $V_{2}$ for different values of $\varepsilon$. 


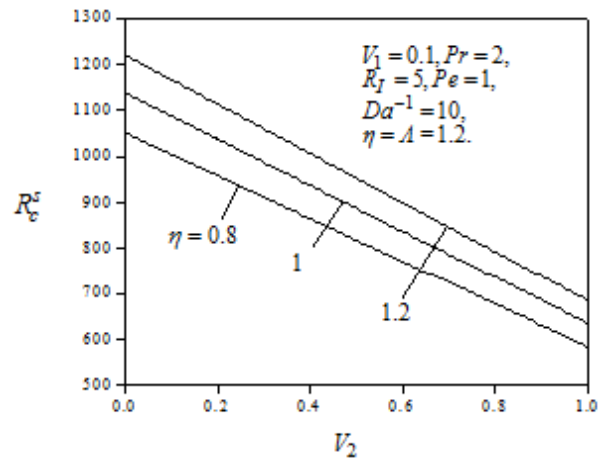

Fig. 10. Plot of $R_{c}^{s}$ Vs. $V_{2}$ for different values of thermal anisotropy parameters $\eta$.

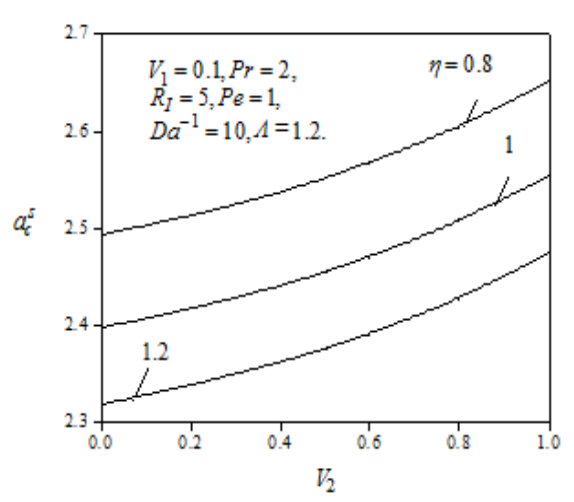

Fig. 11. Plot of $a_{c}^{s}$ Vs. $V_{2}$ for different values of $\eta$.

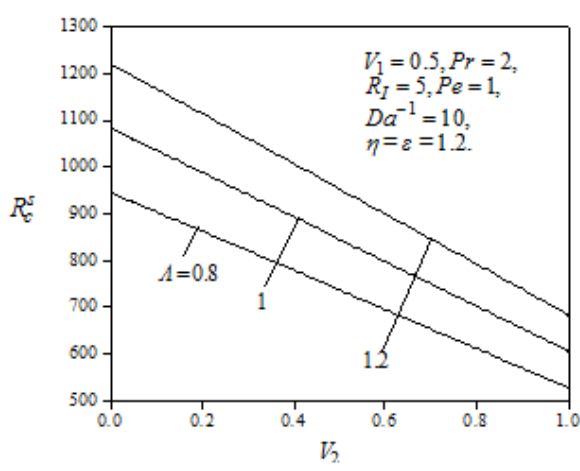

Fig. 12. Plot of $R_{c}^{s}$ Vs. $V_{2}$ for different values of Brinkman number $A$.

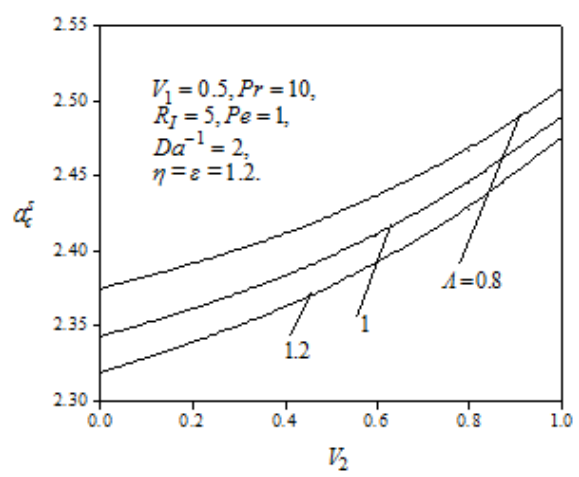

Fig. 13. Plot of $a_{c}^{s}$ Vs. $V_{2}$ for different values of $\Lambda$.

\section{ACKNOWLEDGEMENTS}

The author (RKV) would like to thank Prof. Pradeep, G. Siddheshwar, Department of Mathematics, Bangalore University for suggesting the above problem and for his valuable guidance and support. The author would also like to acknowledge the support and encouragement of the Principal and colleagues in the department at Maharani's Science college for Women, Bangalore -560001 , India.

\section{REFERENCES}

Ansari. A. (2007). Internal heat generation in a tall Cavity filled with a porous medium, J. Porous Media, 10, 585-600.

Barletta, A., di Schio, E.R. and Storesletten, L. (2010). Convective roll instabilities of vertical throughflow with viscous dissipation in a horizontal porous layer. Transp. Porous Med. 81, 461-477.

Bhadauria , B. S. , Hashim, I. and Siddheshwar, P. G. (2013). Study of heat transport in a porous medium under G-jitter and internal heating effects , 96(1), 21-37.

Brevdo, L., Ruderman, S. (2009). On the convection in a porous medium with inclined temperature gradient andvertical throughflow. Part I. Normal modes. Transp. Porous Med. $80,137-151$.

Chandrasekhar, S. (1961). Hydrodynamic and Hydromagnetic stability, London, Oxford University Press.

El-Hakiem, M. A., Rashad, A.M. (2007). Effect of radiation on non-Darcy free convection from a vertical cylinder embedded in a fluid-saturated porous medium with a temperature-dependent viscosity. J. Porous Media,10, 209-218.

Gershuni G. Z. and Zhukhovitskii E. M (1976). Convective stability in incompressible fluids, Jerusalem Keter Publishing House.

Givler, R. C. and Altobelli, A. (1994), A determination of the effective viscosity for Brinkman-Forchheimer model. J. Fluid Mech., 258, 355-367.

Homsy, G. M. and Sherwood, A. E. (1976), Convective stability of incompressible fluids. Israel Program for Scientific Translations, AIChE J., 22 ,168-174.

Jones, M. C. and Persichetti, J. M. (1986). Convective instability in packed beds with through-flow, AIChEJ, $32,1555-1557$.

Khalili A. and Shivakumara I. S. (1998). Onset of convection in a porous layer with net through- 
R. K. Vanishree / JAFM, Vol. 7, No. 4, pp. 581-590, 2014.

flow and internal heat generation, Phys. of fluids, $10,315-317$.

Khalili, A. and Shivakumara, I. S. (2003). NonDarcian effects on the onset of convection in a porous layer with through-flow. Transp. Porous Med., 53 , 245-263.

Khalili, A., Shivakumara, I. S. and Huettel, M. ( 2002), Effects of through-flow and internal heat generation on convective instabilities in an anisotropic porous layer. J. Porous Media, $5,187-198$.

Khalili, A., Shivakumara, I. S. and Suma, S. P. ( 2003). Convective instability in superposed fluid and porous layers with vertical through flow. Transp. Porous Med, 51, 1-18.

Nield, D. A. (1987). Convective instability in porous medium with through-flow, AIChE $J$., $33,1222-1224$.

Nield, D. A., Kuznetsov, A. V. (2011). Onset of convection in a porous medium with strong vertical throughflow, Transp. Porous Med., 90, 883-888.

Shivakumara, I. S. (1999). Boundary and inertia effects on convection in porous media with through-flow, Acta Mech., 137, 151-165.

Shivakumara, I. S. and Nanjundappa, C. E. (2006). Effects of quadratic drag and through-flow on double diffusive convection in a porous layer, Int. Comm. Heat Mass Transfer, 357-363.
Shvartsblat, D. L. (1968). The spectrum of perturbations and convective instability of a plane horizontal fluid layer with permeable boundaries. J. Appl. Mech. (PMM), 32 , 266271.

Shvartsblat, D. L. (1969). Steady convection motions in a plane horizontal fluid layer with permeable boundaries. Fluid Dyn., 4, 54-59.

Siddheshwar, P. G. (1995). Suction-injection effects on Rayleigh-Benard convection in a closely packed porous bed with general boundary condition on temperature, VI Asian Cong. of Fluid Mech., Singapore, 1 590-594.

Sutton F. M. (1970). Onset of convection in a porous channel with net throughflow, Phys. of Fluids, 13, 1931-1942.

Vanishree, R. K., Siddheshwar, P. G. (2010). Effect of rotation on thermal convection in an anisotropic porous mediumwith temperaturedependent viscosity. Transp. Porous Med. 81, 73-87.

Veerraju, N., Srinivasa Babu, K. S. and Rao, C. N. B. (2012). Mixed convection at a vertical plate in a porous medium with magnetic field and variable viscosity, J. Applied Fluid Mech., 5(4), 53-62.

Wooding, R. A. (1960). Rayleigh instability of a thermal boundary layer in flow through porous medium, J. Fluid Mech., 9, 183-192. 